\title{
Evaluation of Anterior Segment Parameters by Pentacam Imaging after Prophylactic Laser Peripheral Iridotomy
}

Ragheb IA, El Shayeb A, Elkhayat MF, Elmohamady MN.

Ophthalmology department- Faculty of Medicine - Benha University

Corresponding author: Islam Abdalla Ragheb, MSc Faculty of Medicine - Benha University. Address: Harameen building, Kholafa' street, Mansoura city, Dakahliya governorate, Egypt. E-mail: dr_islam_abdalla@yahoo.com

Received: 3-1-2021, Accepted: 24-1-2021, Published online:11/3/2021

EJO(MOC) 2021;1:23-33.

Running title: Anterior segment imaging by Pentacam after LPI

\begin{abstract}
Aims: To evaluate the changes in anterior segment parameters after prophylactic laser peripheral iridotomy (LPI) using Pentacam.

Methods and Material: A prospective interventional comparative study. The present study was conducted at Ophthalmology Department, Benha University Hospital in the period from March 2015 to March 2016. Twentyfive primary angle closure suspect (PACS) eyes (group I) and Twenty-five primary angle closure (PAC) eyes and unaffected fellow ones of acute angle closure (AAC-F) (group II) were evaluated with Pentacam before and 1 week after neodymium: yttrium: aluminum garnet (Nd: YAG) LPI. We measured the anterior chamber angle (ACA), central anterior chamber depth (CACD), peripheral anterior chamber depth (PACD), anterior chamber volume (ACV), K readings (K1 and K2), central corneal thickness (CCT) and pupil diameter (PD). Paired t-test was used to investigate the differences in anterior segment parameters before and after LPI. P value of less than or equal (0.05) was considered statistically significant.
\end{abstract}

Results: Fifty eyes in both groups of 35 patients (18 men and 17 women) were included in the study. All subjects were Egyptians. Mean age was 59.4 years old (range 51-70). Mean spherical equivalent was $1.84 \pm 0.58$ diopters (range $1-3.5)$. There was no statistically difference between these groups either in age or in refraction $(\mathrm{P}>0.05)$. In group I , statistically significant differences were found before and 1 week after LPI in mean ACA, PACD, ACV (all $\mathrm{P}<0.05$ ) and statistically insignificant differences were found in mean CACD, PD, CCT and K readings (all $\mathrm{P}>0.05$ ). After LPI, We found ACV increased from $83.88 \pm 4.2 \mathrm{~mm}^{3}$ to $98.36 \pm 7.7 \mathrm{~mm}^{3}$, and also superior, inferior, nasal and temporal PACD increased from $0.97 \mathrm{~mm}, 1.14 \mathrm{~mm}, 0.97 \mathrm{~mm}, 1.29 \mathrm{~mm}$ to $1.10 \mathrm{~mm}, 1.26 \mathrm{~mm}$, $1.11 \mathrm{~mm}$ and $1.41 \mathrm{~mm}$ respectively and ACA increased from $23.3 \pm 2.9^{\circ}$ to $25.45 \pm 2.8^{\circ}$.

In group II , statistically significant differences were found before and 1 week after LPI in ACA, PACD, ACV (all $\mathrm{P}<0.05$ ) and statistically insignificant differences were found in CACD, PD, CCT and $\mathrm{K}$ readings (all $\mathrm{P}>$ 0.05). After LPI, We found ACV increased from $69.8 \pm 10.7 \mathrm{~mm}^{3}$ to $80.72 \pm 14.2 \mathrm{~mm}^{3}$, and also superior, inferior, nasal and temporal PACD increased from $0.98 \mathrm{~mm}, 1.12 \mathrm{~mm}, 0.87 \mathrm{~mm}, 1.2 \mathrm{~mm}$ to $1.12 \mathrm{~mm}, 1.21 \mathrm{~mm}, .96 \mathrm{~mm}$ and $1.33 \mathrm{~mm}$ respectively and ACA increased from $24.1 \pm 2.7^{\circ}$ to $25.8 \pm 2.8^{\circ}$.

Conclusions: The changes of anterior chamber parameters after LPI in both groups can be demonstrated by Pentacam objectively and quantitatively.

Key-words: Laser peripheral iridotomy, Pentacam, primary angle-closure.

Egyptian Journal of Ophthalmology, a publication of Mansoura Ophthalmic Center.

Address: Mansoura Ophthalmic Center, Mansoura University, Mansoura, Egypt.

Website: https://ejomos.journals.ekb.eg

Tel. 0020502202064.

Fax. 0020502202060.

E-mail: ejo@mans.edu.eg 


\section{Introduction:}

Glaucoma is the second cause of blindness worldwide ${ }^{1-2}$. Angle closure glaucoma is a major form of glauco-ma. Several anatomic characteristics including small corneal diameter, shallow central anterior chamber depth (CACD), anterior lens position, excessive lens thickness and short axial length (AL) are considered as predisposing factors for development of angle closure glaucoma ${ }^{3-7}$.

Angle closure glaucoma is classified based on gonioscopy and clinical examination into; Primary angle closure suspect (PACS): characterized by iridotr-abecular contact exceeding $180^{\circ 8-9}$ or $270^{\circ 10}$ but otherwise normal findings; Primary angle closure (PAC): defined as the above mentioned characteristics plus intermittent high intraocular pressure (IOP) or previous attack of angle closure; Primary angle closure glaucoma (PACG): defined as the above mentioned charact-eristics together with glaucomatous optic disc changes and/or visual field defects ${ }^{10}$.

As damage by acute angle closure (AAC) is irreversible, prophylactic laser peripheral iridotomy (LPI) of PACS eyes is crucial. It has also been shown that for patients with a history of AAC attack, without treatment, the risk for developing AAC in the fellow eye is $40-80 \%$ over $5-10$ years $^{11}$. For this reason, development of AAC in one eye is the strongest indication for performing prophylactic LPI in fellow eyes.

The mechanism of LPI is that it creates an opening hole on the peripheral iris to eliminate the pupillary block. Thereby it makes the convex iris flattened which widens the anterior chamber angle ${ }^{12}$.

Pentacam is a noninvasive noncontact method which uses a single rotating Scheimpflug camera ${ }^{13}$. The device generates three dimensional virtual model of the anterior segment. Software allows evaluation and quantification of anterior segment parameters such as central anterior chamber depth
(CACD), peripheral anterior chamber depth (PACD), anterior chamber volume (ACV), pupil diameter (PD) and anterior chamber angle (ACA) of cross section photographs from $0^{\circ}$ to $360^{\circ 14-15}$.

Gonioscopy, anterior segment optical coherence tomography (AS-OCT) and ultrasonic biomicroscopy (UBM) have been used to observe anterior chamber changes. However, gonioscopy is not accurate and AS-OCT doesn't evaluate anterior chamber volume. UBM requires contact and immersion of eyes in the solution which is inconvenient. These methods only obtain cross sections of the anterior segment and cannot provide full three dimensional information of the eye ${ }^{12}$.

Our study aims to examine the impact of prophylactic laser peripheral iridotomy (LPI) on anterior segment parameters using the Pentacam rotating Scheimpflug camera seeking to better evaluation.

\section{Patients and methods:}

This prospective interventional comparative study carried out to assess changes in anterior segments before and one week after prophylactic Nd:YAG laser peripheral iridotomy. The study was conducted at Ophthalmology Department, Benha University Hospital in the period from March 2015 to March 2016. Our study included 50 angle closure eyes of 35 patients indicated for prophylactic laser peripheral iridotomy as in occludable narrow angles and fellow eyes in acute angle closure.

The patients included in the study were Egyptian adults and old ages of both genders with the following criteria: (1) age > 40 years; (2) nonvisibility of the trabecular meshwork for $>180^{\circ}$; (3) no peripheral anterior synechia; (4) tension $<21$ $\mathrm{mmHg}$ without medications; (5) normal optic disc appearance (cup / disc ratio < 0.7). Patients were excluded if they met any of the following criteria: (1) age < 40 years; (2) history of any previous refractive, or ocular surgery and ocular injuries; (3) 
open angle glaucoma, chronic angle closure glaucoma; (4) dense corneal opacities and degenerations; (5) advanced posterior segment problems other than glaucoma.

\section{Complete assessment was done including:}

\section{A) Personal history taking:}

1-Age. 2-Medical history. 3-Past surgical history.

\section{B) Examination data:}

Full ophthalmic examinations were done before LPI and one week after including:

1- Visual acuity using Landolt broken ring chart, then converted to Log MAR for statistical analysis.

2- Intra ocular tension measurement using Goldmann applanation tonometer.

3- Slit lamp biomicroscopy, fundus examination using Volk 90 diopter lens.

4- Gonioscopy using Goldmann triple mirror contact lens.

5- Oculus Pentacam ${ }^{\circledR}$ HR (Oculus Inc, Wetzlar, Germany): to determine anterior chamber parameters such as central anterior chamber depth (CACD), peripheral anterior chamber depth (PACD), anterior chamber angle (ACA), anterior chamber volume (ACV), simulated keratometry readings (Sim K1 and Sim K2 ), central corneal thickness (CCT) and pupil diameter (PD) of cross-section photographs from $0^{\circ}$ to $360^{\circ}$. PACD is defined as $6 \mathrm{~mm}$ from the cornea apex. ACD internal was measured from the endothelium. ACA was measured from horizontal photographs (nasal and temporal).

The patients were classified into two groups:-

Group I: Primary angle closure suspect (PACS).

Group II: Primary angle closure (PAC) \& unaffected fellow eyes of patients with previous attack of primary acute angle closure (AAC-F).
Technique and performance of Pentacam and LPI:

Written informed consents were obtained. In this study we used Oculus Pentacam in a room with dim illumination. The patient was asked not to blink, open his eyes widely and fixate properly. The rotating Scheimpflug camera captured 50 images automatically around the optical axis of the eye. If the quality specification (QS) was not "OK", the examination would be repeated.

One hour before LPI, subjects received one drop of pilocarpine $1 \%$ and one drop of brimonidine tartrate $0.15 \%$. The Nd:YAG laser was used with a single 5-6 mJ pulse at twelve o'clock position of peripheral iris and repeated until patency was achieved. After LPI, subjects received dexamethasone $0.1 \%$ eye drops 4 times for 3 days. One hour after LPI, the IOP was measured and if it was high, timolol $0.5 \%$ eye drops was prescribed twice a day for 7 days. Temporary complications such as increased IOP, minimal iritis and hyphema occurred only in the first hours after LPI. Patients were seen 1 day and 1 week after LPI.

\section{Statistical Analysis}

Data was managed using Statistical Package of Social Sciences (SPSS Inc., Chicago, USA version 20 for Microsoft Window). Statistical significance tests were used. Paired t-test was used to investigate the differences in anterior segment parameters before and after LPI. P value of less than or equal (0.05) was considered statistically significant (at 95\% level of confidence).

Results:

Fifty eyes of 35 patients (18 men and 17 women) were included in the study. All subjects were Egyptians. Mean age of patients was 59.5 years old (range 51-70). Mean spherical equivalent was $1.84 \pm$ 56 dioptres (mean \pm standard deviation). There was no statistical difference between these groups either in age $(P=0.29)$ or in refraction $(P=0.90)$. 


\section{Demographic Study:-}

Primary angle closure suspect (PACS) group(I):-

They included 25 eyes of 16 patients. Their ages ranged between (51-70) years with mean age $(60.08$ \pm 4.80 years) (mean \pm standard deviation). They were 9 males $(56.25 \%)$ and 7 females $(43.75 \%)$ (Table 1). The mean of spherical equivalent (SE) was $1.83 \pm 0.60$ diopters (range 1-2.75) (Table 2).

Primary angle closure (PAC) \& unaffected fellow eyes of patients with previous attack of primary acute angle closure (AAC-F) group(II):-

They included 25 eyes of 19 patients. Their ages ranged between (54-65) years with mean age $(58.88 \square \pm 2.82$ years) (mean \pm SD). They were 9 males (47.37\%) and 10 females (52.63\%) (Table 1). The mean of spherical equivalent (SE) was $1.85 \pm$ 0.55 diopters (range 1-3.5) (Table 2).

Table 1: Comparison of demographic characters of group I (PACS) and group II (PAC and AAC-F) patients.

\begin{tabular}{lccl}
\hline $\begin{array}{l}\text { Demographi } \\
\text { c characters }\end{array}$ & $\begin{array}{c}\text { Group I } \\
\mathbf{N}=\mathbf{2 5}\end{array}$ & $\begin{array}{c}\text { Group II } \\
\mathbf{N}=\mathbf{2 5}\end{array}$ & $\begin{array}{l}\text { Significanc } \\
\mathbf{e}\end{array}$ \\
\hline Age & & & \\
$\begin{array}{l}\text { Mean } \pm \text { SD } \\
(\text { min-max })\end{array}$ & $\begin{array}{c}60.08 \pm 4.80 \\
(51-70)\end{array}$ & $\begin{array}{c}58.88 \pm 2.82 \\
(54-65)\end{array}$ & $\begin{array}{l}\mathrm{t}=1.077 \\
\mathrm{p}=0.288\end{array}$ \\
\hline Gender & & & \\
Male & $9(56.25 \%)$ & $9(47.37 \%)$ & \\
Female & $7(43.75 \%)$ & $10(52.63 \%)$ &
\end{tabular}

PACS $=$ Primary angle closure suspect, $\mathrm{PAC}=$ Primary angle closure, AAC-F=Acute angle closure fellow eyes, $\mathrm{SD}=$ Standard deviation. $\mathrm{t}$ for independent $\mathrm{t}$ test.

Table 2: Comparison between refractive power of group I (PACS) and group II (PAC and AAC-F) patients.

\begin{tabular}{lccl}
\hline Refraction & $\begin{array}{c}\text { Group I } \\
\mathbf{N}=\mathbf{2 5}\end{array}$ & $\begin{array}{c}\text { Group II } \\
\mathbf{N}=\mathbf{2 5}\end{array}$ & Significance \\
\hline $\mathrm{SE}$ & & & \\
Mean $\pm \mathrm{SD}$ & $1.83 \pm$ & $1.85 \pm$ & $\mathrm{t}=0.123$ \\
$($ min-max $)$ & 0.60 & 0.55 & $\mathrm{p}=0.903$ \\
& $(1-2.75)$ & $(1-3.5)$ & \\
\hline
\end{tabular}

PACS $=$ Primary angle closure suspect, $\mathrm{PAC}=$ Primary angle closure, AAC-F=Acute angle closure fellow eyes, $\mathrm{SE}=$ Spherical equivalent, $\mathrm{SD}=$ Standard deviation. $\mathrm{t}$ for independent $\mathrm{t}$ test.

\section{Ocular Study:-}

Pre-LPI anterior segment Findings measured by

\section{Pentacam:-}

Table No. (3) shows comparison of anterior segment parameters of group I and II patients before laser peripheral iridotomy using independent samples t-test. There was statistically significant difference among both groups in some parameters such as ACV, nasal PACD, CACD, PD and $\mathrm{K}$ readings. On the other hand, there was no statistically significant difference among both groups in other parameters such as ACA, superior PACD, inferior PACD, temporal PACD and CCT. There was positive correlation between CACD and $\operatorname{ACV}(\mathrm{R}=0.57)$.

Table 3: Comparison of anterior segment parameters of group I and II patients before laser peripheral iridotomy.

\begin{tabular}{|c|c|c|c|}
\hline $\begin{array}{l}\text { Morphology } \\
\text { parameters }\end{array}$ & $\begin{array}{c}\text { Group I } \\
\mathbf{N}=25 \\
(\text { Mean } \pm \\
\text { SD) }\end{array}$ & $\begin{array}{c}\text { Group II } \\
\text { N=25 } \\
\text { (Mean } \pm \\
\text { SD) }\end{array}$ & Significance \\
\hline $\mathrm{ACV}$ & $83.88 \pm 4.22$ & $69.8 \pm 10.77$ & $\begin{array}{l}\mathrm{t}=6.089 \\
\mathrm{p}<0.001 *\end{array}$ \\
\hline $\mathrm{ACA}$ & $23.32 \pm 2.94$ & $24.17 \pm 2.77$ & $\begin{array}{l}\mathrm{t}=1.05 \\
\mathrm{p}=0.299\end{array}$ \\
\hline PACD & & & \\
\hline Superior & $0.978 \pm 0.102$ & $0.986 \pm 0.236$ & $\begin{array}{l}\mathrm{t}=0.171 \\
\mathrm{p}=0.865\end{array}$ \\
\hline Inferior & $1.145 \pm 0.131$ & $1.122 \pm 0.151$ & $\begin{array}{l}\mathrm{t}=0.569 \\
\mathrm{p}=0.572\end{array}$ \\
\hline Nasal & $0.975 \pm 0.125$ & $0.871 \pm 0.11$ & $\begin{array}{l}\mathrm{t}=3.109 \\
\mathrm{p}=0.003^{*}\end{array}$ \\
\hline Temporal & $1.294 \pm 0.121$ & $1.207 \pm 0.195$ & $\begin{array}{l}t=1.871 \\
p=0.067\end{array}$ \\
\hline CACD & $1.918 \pm 0.149$ & $1.824 \pm 0.112$ & $\begin{array}{l}\mathrm{t}=2.524 \\
\mathrm{p}=0.015^{*}\end{array}$ \\
\hline PD & $2.63 \pm 0.39$ & $2.989 \pm 0.67$ & $\begin{array}{l}\mathrm{t}=2.292 \\
\mathrm{p}=0.026^{*}\end{array}$ \\
\hline $\mathrm{CCT}$ & $521.04 \pm 16.49$ & $517.56 \pm 27.82$ & $\begin{array}{l}\mathrm{t}=0.538 \\
\mathrm{p}=0.593\end{array}$ \\
\hline k1 & $42.23 \pm 1.67$ & $43.23 \pm 1.67$ & $\begin{array}{l}\mathrm{t}=2.113 \\
\mathrm{p}=0.04^{*}\end{array}$ \\
\hline k2 & $43.55 \pm 1.47$ & $44.42 \pm 1.26$ & $\begin{array}{l}\mathrm{t}=2.237 \\
\mathrm{p}=0.03^{*}\end{array}$ \\
\hline \multicolumn{4}{|c|}{$\begin{array}{l}\mathrm{PACS}=\text { Primary angle closure suspect, } \mathrm{PAC}=\text { Primary } \\
\text { angle closure, AAC-F=Acute angle closure fellow eyes, } \\
\mathrm{SD}=\text { Standard deviation, } \mathrm{ACV}=\text { Anterior chamber volume, } \\
\mathrm{ACA}=\text { Anterior chamber angle, } \mathrm{PACD}=\text { Peripheral anterior } \\
\text { chamber depth, } \mathrm{CACD}=\text { Central anterior chamber depth, }\end{array}$} \\
\hline
\end{tabular}


$\mathrm{PD}=$ Pupil diameter, $\mathrm{CCT}=\mathrm{Central}$ corneal thickness, $\mathrm{K} 1=$ Keratometry reading $1, \mathrm{~K} 2=$ Keratometry reading 2 . $\mathrm{t}$ for independent $\mathrm{t}$ test.

* $\mathrm{P}<0.05$ was considered to be statistically significant.

Post-LPI anterior segment Findings measured by Pentacam:-

Table No. (4) shows comparison of anterior segment parameters of group I and II patients after laser peripheral iridotomy using independent samples t-test. There was statistically significant difference among both groups in some parameters such as ACV, nasal PACD, CACD and PD. On the other hand, there was no statistically significant difference among both groups in other parameters such as ACA, superior PACD, inferior PACD, temporal $\mathrm{PACD}, \mathrm{CCT}$ and $\mathrm{K}$ readings. ACV, ACA and PACD showed increase more than their pre-LPI values in the two groups of the study.

Table 4: Comparison of anterior segment parameters of group I and II patients after laser peripheral iridotomy.

\begin{tabular}{|c|c|c|c|c|c|c|c|}
\hline & \multirow{2}{*}{ parameters } & \multicolumn{2}{|c|}{$N=25$} & \multirow[b]{2}{*}{ Significance } \\
\hline $\begin{array}{l}\text { Morphology } \\
\text { parameters }\end{array}$ & $\begin{array}{c}\text { Group I } \\
\mathbf{N}=25 \\
(\text { Mean } \pm \text { SD) } \\
\end{array}$ & $\begin{array}{c}\text { Group II } \\
\text { N=25 } \\
(\text { Mean } \pm \text { SD) } \\
\end{array}$ & Significance & & $\begin{array}{c}\text { Before LPI } \\
(\text { Mean } \pm \text { SD) }\end{array}$ & $\begin{array}{c}\text { After LPI } \\
\text { (Mean } \pm \\
\text { SD) }\end{array}$ & \\
\hline $\mathrm{ACV}$ & $98.36 \pm 7.76$ & $80.72 \pm 14.26$ & $\begin{array}{l}\mathrm{t}=5.433 \\
\mathrm{p}<0.001 *\end{array}$ & $\mathrm{ACV}$ & $83.88 \pm 4.22$ & $98.36 \pm 7.76$ & $\begin{array}{l}\mathrm{t}=13.579 \\
\mathrm{p}<0.001^{*}\end{array}$ \\
\hline $\mathrm{ACA}$ & $25.46 \pm 2.89$ & $25.85 \pm 2.83$ & $\begin{array}{l}t=0.484 \\
p=0.631\end{array}$ & ACA & $23.32 \pm 2.94$ & $25.46 \pm 2.89$ & $\begin{array}{l}t=13.66 \\
p<0.001 *\end{array}$ \\
\hline PACD & & & & PACD & & & \\
\hline Superior & $1.104 \pm 0.097$ & $1.122 \pm 0.344$ & $\begin{array}{l}\mathrm{t}=0.246 \\
\mathrm{p}=0.807\end{array}$ & Superior & $0.978 \pm 0.102$ & $1.104 \pm 0.097$ & $\begin{array}{l}\mathrm{t}=8.165 \\
\mathrm{p}<0.001 *\end{array}$ \\
\hline Inferior & $1.266 \pm 0.12$ & $1.214 \pm 0.176$ & $\begin{array}{l}\mathrm{t}=1.219 \\
\mathrm{p}=0.229\end{array}$ & Inferior & $1.145 \pm 0.131$ & $1.266 \pm 0.12$ & $\begin{array}{l}\mathrm{t}=14.341 \\
\mathrm{p}<0.001 *\end{array}$ \\
\hline Nasal & $1.114 \pm 0.124$ & $0.969 \pm 0.13$ & $\begin{array}{l}\mathrm{t}=3.995 \\
\mathrm{p}<0.001^{*}\end{array}$ & Nasal & $0.975 \pm 0.125$ & $1.114 \pm 0.124$ & $\begin{array}{l}\mathrm{t}=13.449 \\
\mathrm{p}<0.001^{*}\end{array}$ \\
\hline Temporal & $1.418 \pm 0.137$ & $1.33 \pm 0.227$ & $\begin{array}{l}t=1.613 \\
p=0.113\end{array}$ & Temporal & $1.294 \pm 0.121$ & $1.418 \pm 0.137$ & $\begin{array}{l}t=14.751 \\
p<0.001^{*}\end{array}$ \\
\hline CACD & $1.927 \pm 0.166$ & $1.84 \pm 0.119$ & $\begin{array}{l}\mathrm{t}=2.052 \\
\mathrm{p}=0.046^{*}\end{array}$ & CACD & $1.918 \pm 0.149$ & $1.927 \pm 0.166$ & $\begin{array}{l}t=1.709 \\
p=0.1\end{array}$ \\
\hline PD & $2.64 \pm 0.39$ & $3.036 \pm 0.75$ & $\begin{array}{l}\mathrm{t}=2.324 \\
\mathrm{p}=0.024^{*}\end{array}$ & PD & $2.63 \pm 0.39$ & $2.64 \pm 0.39$ & $\begin{array}{l}t=0.276 \\
p=0.785\end{array}$ \\
\hline CCT & $521.92 \pm 21.197$ & $516.56 \pm 25.863$ & $\begin{array}{l}\mathrm{t}=0.801 \\
\mathrm{p}=0.427\end{array}$ & $\mathrm{CCT}$ & $521.04 \pm 16.49$ & $521.92 \pm 21.197$ & $\begin{array}{l}\mathrm{t}=0.41 \\
\mathrm{p}=0.685\end{array}$ \\
\hline k1 & $42.33 \pm 1.645$ & $43.11 \pm 1.51$ & $\begin{array}{l}\mathrm{t}=1.748 \\
\mathrm{p}=0.087\end{array}$ & k1 & $42.23 \pm 1.67$ & $42.33 \pm 1.645$ & $\begin{array}{l}\mathrm{t}=0.844 \\
\mathrm{p}=0.407\end{array}$ \\
\hline k2 & $43.43 \pm 1.431$ & $44.16 \pm 1.39$ & $\begin{array}{l}\mathrm{t}=1.832 \\
\mathrm{p}=0.073\end{array}$ & $\mathrm{k} 2$ & $43.55 \pm 1.47$ & $43.43 \pm 1.431$ & $\begin{array}{l}\mathrm{t}=1.492 \\
\mathrm{p}=0.149\end{array}$ \\
\hline $\begin{array}{l}\mathrm{PACS}=\mathrm{P} \\
\text { angle clos } \\
\mathrm{SD}=\text { Stanc }\end{array}$ & $\begin{array}{l}\text { nary angle closu } \\
\text { e, AAC-F=Acute } \\
\text { d deviation, ACV }\end{array}$ & re suspect, $\mathrm{PAC}=$ & $\begin{array}{l}\text { Primary } \\
\text { low eyes, } \\
\text { r volume, }\end{array}$ & $\begin{array}{l}\mathrm{PACS}=\text { Prin } \\
\text { peripheral } \\
\mathrm{ACV}=\text { Anteric }\end{array}$ & $\begin{array}{l}\mathrm{y} \text { angle closu } \\
\text { dotomy, } \mathrm{SD}= \\
\text { chamber volum }\end{array}$ & $\begin{array}{l}\text { re suspect, LPI } \\
=\text { Standard } \\
\text {, } \mathrm{ACA}=\text { Anterior }\end{array}$ & $\begin{array}{l}=\text { Laser } \\
\text { deviation, } \\
\text { chamber }\end{array}$ \\
\hline
\end{tabular}


angle, $\mathrm{PACD}=$ Peripheral anterior chamber depth, $\mathrm{CACD}=$ Central anterior chamber depth, $\mathrm{PD}=$ Pupil diameter, $\mathrm{CCT}=\mathrm{Central}$ corneal thickness, $\mathrm{K} 1=$ Keratometry reading $1, \mathrm{~K} 2=$ Keratometry reading 2 .

$\mathrm{t}$ for paired $\mathrm{t}$ test.

* $\mathrm{P}<0.05$ was considered to be statistically significant.

Example: Male patient aged 51 years from Benha. He had no medical or surgical history. His refraction was compound hypermetrope. His best corrected visual acuity (BCVA) was 6/6 in both eyes. Right eye Oculus Pentacam picture before LPI (Figure 1). The patient was diagnosed as PACS and LPI was performed to him. Right eye Oculus Pentacam picture one week after LPI (Figure 2).

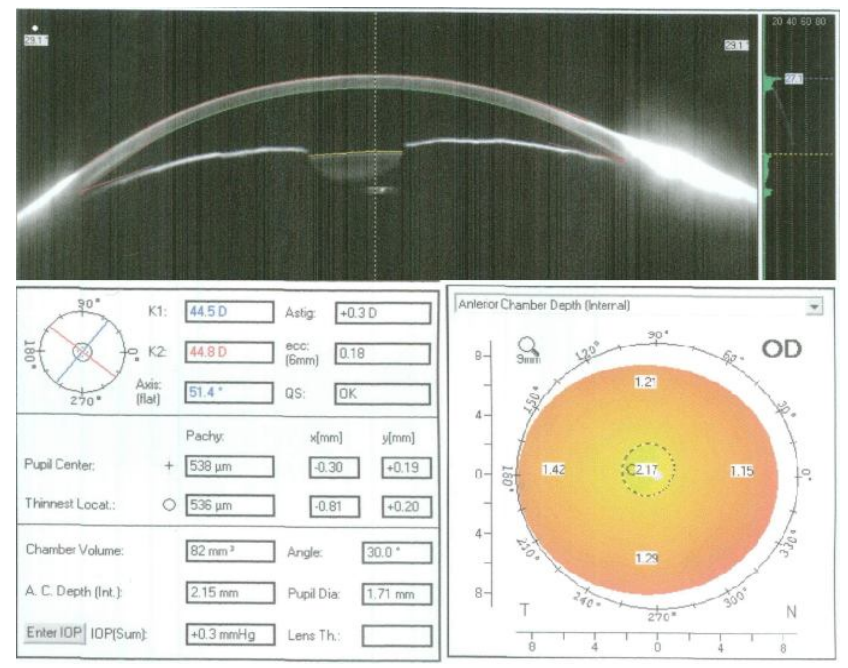

Figure 1: Right eye Oculus Pentacam picture before LPI.

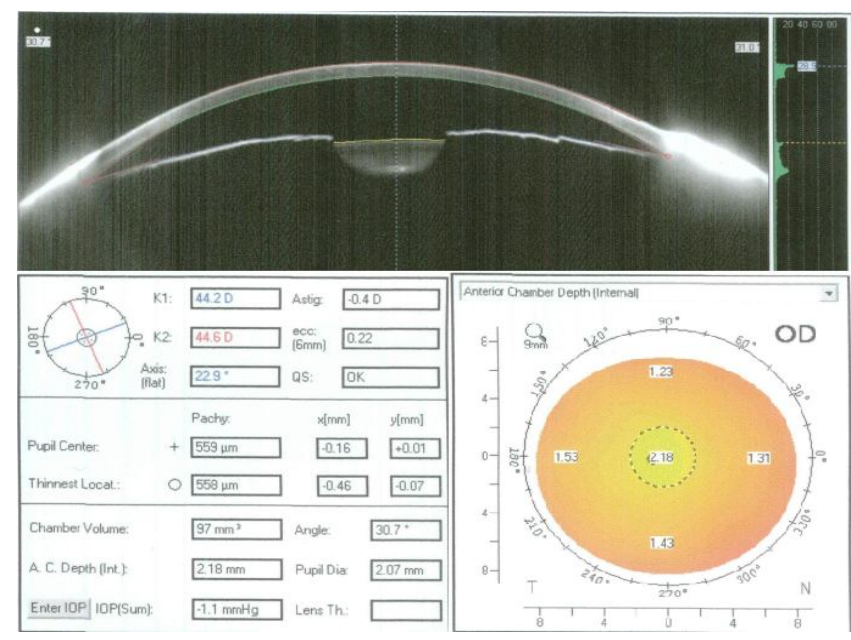

Figure 2: Right eye Oculus Pentacam picture after LPI.

\section{In group II (PAC and AAC-F):-}

Table No. (6) shows comparison of anterior segment parameters of group II patients before and after laser peripheral iridotomy using paired t test.
There was statistically significant difference before and after LPI in ACV, ACA and PACD parameters ( $\mathrm{P}$ value $<0.05$ ). On the other hand, there was no statistically significant difference before and after LPI in CACD, PD, CCT and $K$ readings ( $P$ value $>0.05)$.

Example: Male patient aged 54 years from Dakahliya. He sought medical advice as he was complaining of headache. He had history of acute attack of AAC in right eye. His refraction was simple hypermetrope. His best corrected visual acuity (BCVA) was 6/60 in right eye and 6/6 in left eye. Left eye Oculus Pentacam picture before LPI (Figure 3). The patient was diagnosed as right AAC and LPI was performed to the fellow eye (left eye). Left eye Oculus Pentacam picture one week after LPI (Figure 4).

Table 6: Comparison of anterior chamber morphology in PAC and AAC-F patients (group II) before and after laser iridotomy.

\begin{tabular}{|c|c|c|c|}
\hline \multirow[t]{2}{*}{$\begin{array}{l}\text { Morphology } \\
\text { parameters }\end{array}$} & \multicolumn{2}{|c|}{$\begin{array}{c}\text { Group II } \\
\mathrm{N}=25\end{array}$} & \multirow[t]{2}{*}{ Significance } \\
\hline & $\begin{array}{l}\text { Before LPI } \\
(\text { Mean } \pm \text { SD) }\end{array}$ & $\begin{array}{c}\text { After LPI } \\
(\text { Mean } \pm \text { SD) }\end{array}$ & \\
\hline $\mathrm{ACV}$ & $69.8 \pm 10.77$ & $80.72 \pm 14.26$ & $\begin{array}{l}\mathrm{t}=6.554 \\
\mathrm{p}<0.001 *\end{array}$ \\
\hline ACA & $24.17 \pm 2.77$ & $25.85 \pm 2.83$ & $\begin{array}{l}\mathrm{t}=9.372 \\
\mathrm{p}<0.001 *\end{array}$ \\
\hline \multicolumn{4}{|l|}{ PACD } \\
\hline Superior & $0.986 \pm 0.236$ & $1.122 \pm 0.344$ & $\begin{array}{l}\mathrm{t}=3.283 \\
\mathrm{p}=0.003 *\end{array}$ \\
\hline Inferior & $1.122 \pm 0.151$ & $1.214 \pm 0.176$ & $\begin{array}{l}t=5.173 \\
p<0.001 *\end{array}$ \\
\hline Nasal & $0.871 \pm 0.11$ & $0.969 \pm 0.13$ & $\begin{array}{l}t=4.757 \\
p<0.001 *\end{array}$ \\
\hline Temporal & $1.207 \pm 0.195$ & $1.33 \pm 0.227$ & $\begin{array}{l}\mathrm{t}=5.269 \\
\mathrm{p}<0.001 *\end{array}$ \\
\hline CACD & $1.824 \pm 0.112$ & $1.84 \pm 0.119$ & $\begin{array}{l}t=1.672 \\
p=0.108\end{array}$ \\
\hline PD & $2.989 \pm 0.67$ & $3.036 \pm 0.75$ & $\begin{array}{l}\mathrm{t}=1.104 \\
\mathrm{p}=0.281\end{array}$ \\
\hline CCT & $\begin{array}{c}517.56 \pm \\
27.82\end{array}$ & $\begin{array}{c}516.56 \pm \\
25.86\end{array}$ & $\begin{array}{l}t=0.525 \\
p=0.604\end{array}$ \\
\hline $\mathrm{k} 1$ & $43.23 \pm 1.67$ & $43.11 \pm 1.51$ & $\begin{array}{l}\mathrm{t}=0.966 \\
\mathrm{p}=0.344\end{array}$ \\
\hline $\mathrm{k} 2$ & $44.42 \pm 1.26$ & $44.16 \pm 1.39$ & $\begin{array}{l}t=1.857 \\
p=0.076\end{array}$ \\
\hline
\end{tabular}

$\mathrm{PACS}=$ Primary angle closure suspect, $\mathrm{PAC}=$ Primary angle closure, AAC-F=Acute angle closure fellow eyes, $\mathrm{LPI}=$ Laser peripheral iridotomy, $\mathrm{SD}=\mathrm{Standard}$ deviation, $\mathrm{ACV}=$ Anterior chamber volume, $\mathrm{ACA}=$ Anterior chamber 
angle, PACD=Peripheral anterior chamber depth, $\mathrm{CACD}=$ Central anterior chamber depth, $\mathrm{PD}=$ Pupil diameter, $\quad \mathrm{CCT}=$ Central corneal thickness, $\mathrm{K} 1=$ Keratometry reading $1, \mathrm{~K} 2=$ Keratometry reading 2 . $\mathrm{t}$ for paired $\mathrm{t}$ test.

* $\mathrm{P}<0.05$ was considered to be statistically significant.

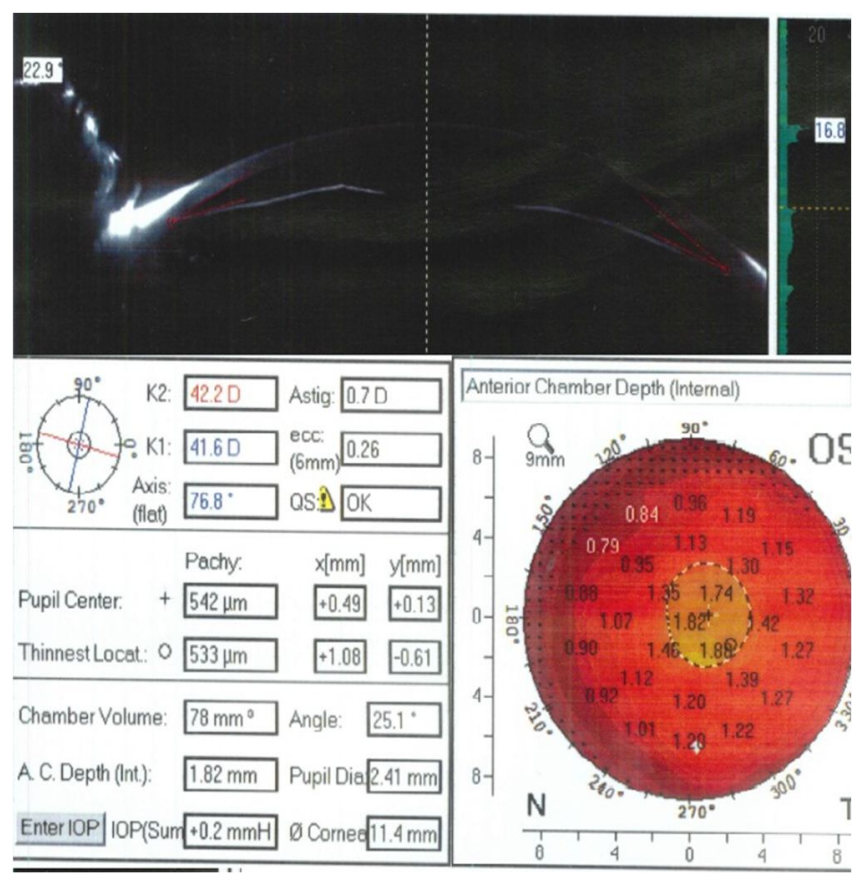

Figure 3: Left eye Oculus Pentacam picture before LPI.
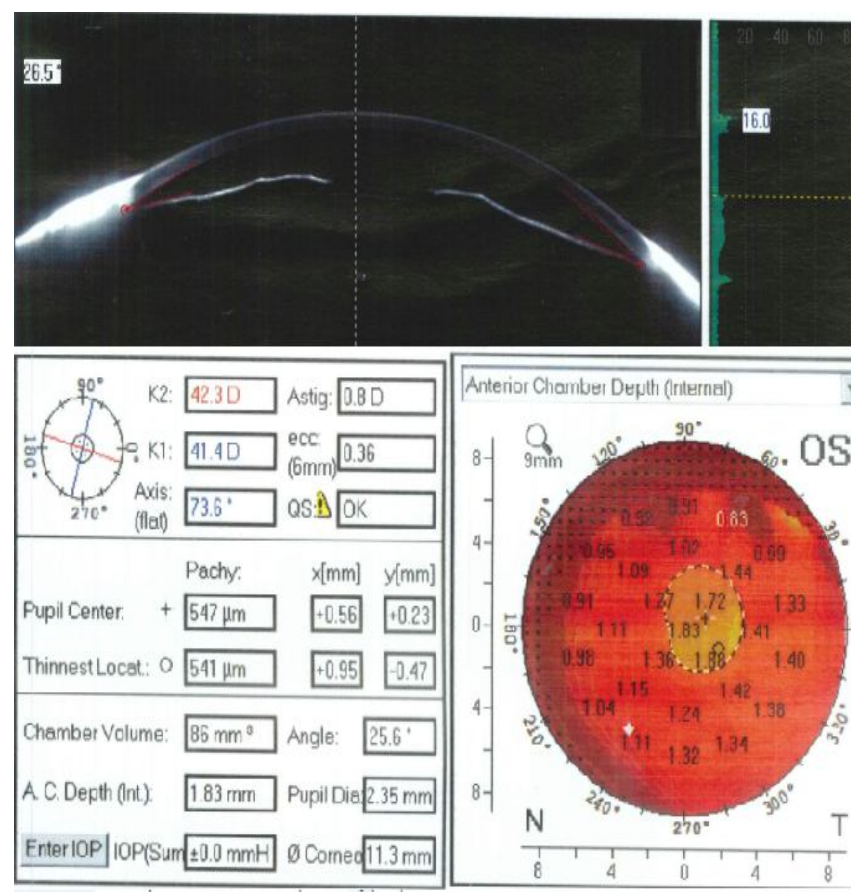

Figure 4: Left eye Oculus Pentacam picture after LPI.

\section{Discussion:}

This study aimed to evaluate the efficacy of LPI as a prophylactic maneuver in the management of the patients with occludable narrow angles using
Pentacam. LPI has proved to be successful in preventing the development of PACS and PAC into $\mathrm{PACG}^{16-17}$. The study results confirmed the effectiveness of LPI, as there were significant increases in ACV, ACA and PACD after LPI in both groups.

Pentacam generates a 3-D virtual model of the anterior segment, $\mathrm{ACV}$ is a unique parameter which other anterior segment devices could not give. In our study, ACV in group I (PACS) increased more greatly than in group II (PAC and AAC), thus we assume that LPI might be more effective in PACS patients.

In this study, in group I, mean ACV increased from $83.9 \mathrm{~mm}^{3}$ to $98.4 \mathrm{~mm}^{3}(\mathrm{P}<0.001)$. In group II, mean ACV increased from $69.8 \mathrm{~mm}^{3}$ to $80.7 \mathrm{~mm}^{3}$ (P $<0.001)$.

Similar to our study, Esmaeili et al. (2013) reported that mean ACV increased sharply after LPI in PACS from $85.97 \mathrm{~mm}^{3}$ to $99.25 \mathrm{~mm}^{3}(\mathrm{P}<0.001)^{14}$. Lee et al. (2011) observed that mean ACV increased significantly after the LPI in PACS from $79.96 \mathrm{~mm}^{3}$ to $95.63 \mathrm{~mm}^{3}(\mathrm{P}<0.001)^{18}$. Li et al. (2014) found that there was statistically significant difference before and 1 week after LPI as mean ACV increased from $58.2 \mathrm{~mm}^{3}$ to $83.66 \mathrm{~mm}^{3}$ in PAC patients $(\mathrm{P}<$ $0.001)^{19}$.

Talajic et al. study and Vryonis et al. study (2013) agreed with our study. They concluded that mean ACV increased significantly in PACS patients after LPI ${ }^{20-21}$. Jain et al. study (2012) and Wu et al. study (2010) showed that mean ACV increased significantly after iridotomy in PAC patients ${ }^{22-23}$.

In our study, ACA was measured as average of nasal and temporal angles, as they are more accurate to be measured by Pentacam. Although Pentacam is an accurate method in anterior segment imaging, the inability of the Pentacam to visualize the most peripheral part of the iris and the base of the ACA might justify this observation that ACA is unreliable 
and mostly overestimated in eyes with a narrow $\mathrm{ACA}^{24-25}$.

In our study, in group I, mean ACA increased from $23.32 \pm 2.94^{\circ}$ to $25.46 \pm 2.89^{\circ}(\mathrm{P}<0.001)$. In group II, mean ACA increased from $24.17 \pm 2.77^{\circ}$ to $25.85 \pm 2.83^{\circ}(\mathrm{P}<0.001)$.

On one hand, Esmaeili et al. (2013) reported that the mean ACA increased significantly in PACS patients after $\mathrm{LPI}^{14}$. On the other hand, Lee et al. (2011) observed that the mean ACA increased insignificantly in PACS patients after $\mathrm{LPI}^{18}$. Also Li et al. (2014) found that there were statistically significant differences in PAC patients after LPI in nasal and temporal ACA $(\mathrm{P}<0.05)$ except for superior $(\mathrm{P}=0.053)$ and inferior ACA $(\mathrm{P}=0.389)^{19}$.

Talajic et al. study and Vryonis et al. study (2013) agreed with our study. They concluded that mean ACA increased significantly after LPI in PACS patients ${ }^{20-21}$.

In our study, we measured PACD as $6 \mathrm{~mm}$ from the cornea apex, as this area coincided with maximal iris bombé elevation in most of cases and was more nearer to iridotomy site. Lee et al. (2011) study defined PACD as 4,6 , and $8 \mathrm{~mm}$ from eye center ${ }^{18}$. Jain et al. (2012) study defined PACD as 4 and 8 $\mathrm{mm}$ from cornea apex ${ }^{22}$. On the contrary, Li et al. (2014) defined PACD as $4 \mathrm{~mm}$ from cornea apex but this parameter became more to the center than to the periphery of the iris and this was more far away from the iridotomy site ${ }^{19}$.

In our study, the mean superior, inferior, nasal and temporal PACD in both groups increased significantly. Similar to our study, significant PACD deepening was observed after LPI in all meridians in Lee et al. (2011), Li et al. (2014), Jain et al. (2012), Wu et al. (2010) and Antoniazzi et al. (2010) studies (all $\mathrm{P}<0.01)^{18-19,22-23,26}$.

The results of the present study showed insignificant change in CACD after the LPI in both group I $(p=0.1)$ and group II $(p=0.108)$, agreeing with Esmaeili et al. (2013) $(\mathrm{P}=0.09)$, Lee et al. (2011) $(\mathrm{P}=0.526), \mathrm{Li}$ at al. (2014) $(\mathrm{P}=0.453)$, Vryonis et al. (2013) $(\mathrm{P}=0.49)$ and Jain et al. (2012) studies. The LPI moves the iris toward the posterior chamber, however, the location of the lens is not affected; thus may not affect the CACD ${ }^{14,18-19,21-22}$.

Our study showed that PD did not change significantly after LPI in both group $I(p=0.785)$ and group II $(p=0.281)$. PD could affect the measurements of anterior chamber parameters. In order to overcome this problem, we tried to control the lighting and fixation to ensure that PD remained the same in 2 sessions of the measurements ${ }^{27}$.

Similar to our study, Esmaeili et al. (2013) study showed insignificant change in PD after LPI $(\mathrm{P}=0.33)$ and the same results were with Talajic et al. (2013) and $\mathrm{Li}$ et al. (2014) studies $(\mathrm{P}=$ $0.221)^{14,19-20}$.

Pakravan et al. (2012) compared between AAC (group I), PACS (group II) and normal persons (group III). They concluded that Eyes with anterior chamber volume $\leq 100 \mathrm{~mm}^{3}$, depth $\leq 2.1 \mathrm{~mm}$ and angle $\leq 26^{\circ}$ may be considered at high risk for developing AAC. These criteria could be helpful for making decisions regarding prophylactic LPI ${ }^{28}$.

Zou et al. (2010) found that ACD, ACV and ACA decrease mildly with age. They reported that anterior chamber parameters of PAC are smaller than that of normal eyes, thus prophylactic LPI was indicated $^{29}$.

Wang et al. (2002) stated that $55 \%$ of all angleclosure in China was caused by multiple mechanisms, with only $38 \%$ was pure pupil block ${ }^{30}$. He et al. (2006) reported that the dark-prone provocative test was positive in $60 \%$ of Taiwanese Chinese eyes after iridectomy, compared with $12.5 \%$ in normal eyes ${ }^{31}$.

Our study also confirmed that even though pupil block was eliminated, PACS and PAC patients still had much smaller ACA, ACD and ACV, which 
indicates multiple mechanisms for angle closure exist. So even after conventional YAG laser iridotomy, PAC patients need routine follow up to prevent progression of angle closure. For some of these structural variations, other preventive treatments may need to be devised such as laser iridoplasty ${ }^{18}$.

\section{Conclusions:}

Pentacam has unsurpassed ability to measure and visualize the anterior segment. Pentacam is of great utility in daily clinical practice and provides a wealth of information. The changes of anterior chamber parameters after LPI in both groups can be demonstrated by Pentacam objectively and quantitatively. PACS patients had more significant changes in ACA, ACV and PACD in comparison to PAC patients after LPI. Two thirds of PACS continued to have two quadrants of non-visible trabecular meshwork, possibly due to non-pupillary block mechanism of angle closure.

\section{Declarations:}

\section{Acknowledgements}

The authors would like to thank all the employees who agreed to participate in this study and all the patients involved in this study for their support.

\section{Funding: Nil.}

Authors' Contributions: MN Elmohamady and A El Shayeb analyzed and interpreted the patient data. IA Ragheb performed the patient examination, data collection and was a major contributor in writing the manuscript. All authors read and approved the final manuscript.

Competing interests: The authors have no proprietary or commercial interest in any materials discussed in this article.

Ethics approval and consent to participate: The study was approved by the ethics committee of Benha University, and the ethics committee of ophthalmology department in Benha university hospitals, Egypt. Informed consent obtained from all study participants was written.

\section{Prior publication: Nil.}

Availability of data and materials: The data that support the findings of this study are available upon request from the corresponding author [IA Ragheb]. The data are not publicly available due to containing information that could compromise the privacy of research participants. Data are available from the authors upon reasonable request.

Abbreviations: PACS: primary angle closure suspect; PAC: primary angle closure; PACG: primary angle closure glaucoma; AAC-F: unaffected fellow eye of acute angle closure; Nd: YAG: neodymium: yttrium: aluminum garnet; ACA: anterior chamber angle; $\mathrm{CACD}$ : central anterior chamber depth; PACD: peripheral anterior chamber depth; $\mathrm{ACV}$ : anterior chamber volume; $\mathrm{K}$ readings: keratometry readings; $\mathrm{CCT}$ : central corneal thickness; PD: pupil diameter; LPI: laser peripheral iridotomy; PAS: peripheral anterior synechia; BCVA: best corrected visual acuity; IOP: intraocular pressure.

No financial interest

No conflict of interests

\section{References:}

1. Quigley HA, Broman AT. The number of people with glaucoma worldwide in 2010 and 2020. Br J Ophthalmol 2006;90:262-7.

2. Thylefors B, Negrel AD, Pararajasegaram R, Dadzie KY. Global data on blindness. Bull World Health Organ 1995;73:115-21.

3. Alsbirk PH. Primary angle-closure glaucoma. Oculometry, epidemiology, and genetics in a high risk population. Acta Ophthalmol Suppl 1976:5-31.

4. Lowe RF. Aetiology of the anatomical basis for primary angle-closure glaucoma. Biometrical comparisons between normal eyes and eyes with 
primary angle-closure glaucoma. $\mathrm{Br} \quad \mathrm{J}$ Ophthalmol 1970;54:161-9.

5. Sihota R, Lakshmaiah NC, Agarwal HC, Pandey RM, Titiyal JS. Ocular parameters in the subgroups of angle closure glaucoma. Clin Experiment Ophthalmol 2000;28:253-8.

6. George R, Paul PG, Baskaran M, Ramesh SV, Raju P, Arvind H, McCarty C, Vijaya L. Ocular biometry in occludable angles and angle closure glaucoma: a population based survey. $\mathrm{Br} \mathrm{J}$ Ophthalmol 2003;87:399-402.

7. Lan YW, Hsieh JW, Hung PT. Ocular biometry in acute and chronic angle-closure glaucoma. Ophthalmologica 2007;221:388-94.

8. Thomas R, George R, Parikh R, Muliyil J, Jacob A. Five year risk of progression of primary angle closure suspects to primary angle closure: a population based study. $\mathrm{Br} \mathrm{J}$ Ophthalmol 2003;87:450-4.

9. Thomas R, Parikh R, Muliyil J, Kumar RS. Fiveyear risk of progression of primary angle closure to primary angle closure glaucoma: a populationbased study. Acta Ophthalmol Scand 2003;81:480-5.

10.Foster PJ, Buhrmann R, Quigley HA, Johnson GJ. The definition and classification of glaucoma in prevalence surveys. $\mathrm{Br} \mathrm{J}$ Ophthalmol 2002;86:238-42.

11.American Academy of Ophthalmology. Glaucoma. 2011-2012: Sección 10. In: Cioffi GA, editor. American Academy of Ophthalmology. Spain: Elsevier Health Sciences; 2012.

12.He M, Friedman DS, Ge J, Huang W, Jin C, Cai X, Khaw PT, Foster PJ. Laser peripheral iridotomy in eyes with narrow drainage angles: ultrasound biomicroscopy outcomes. The Liwan Eye Study. Ophthalmology 2007;114:1513-19.

13. Rabsilber TM, Khoramnia R, Auffarth GU. Anterior chamber measurements using Pentacam rotating Scheimpflug camera. J Cataract Refract Surg 2006;32:456-9.

14. Esmaeili A, Barazandeh B, Ahmadi S, Haghi A, Ahmadi Hosseini SM, Abolbashari F. Assessment of the anterior chamber parameters after laser iridotomy in primary angle close suspect using Pentacam and gonioscopy. Int $\mathrm{J}$ Ophthalmol 2013;6:680-4.

15. Gupta M, Ram J, Jain A, Sukhija J, Chaudhary M. Correlation of nuclear density using the Lens Opacity Classification System III versus Scheimpflug imaging with phacoemulsification parameters. J Cataract Refract Surg 2013;39:1818-23.

16. Johnson G, Foster P. Can we prevent angleclosure glaucoma? Eye 2005;19:1119-24.

17. Pandav SS, Kaushik S, Jain R, Bansal R, Gupta A. Laser peripheral iridotomy across the spectrum of primary angle closure. Can J Ophthalmol 2007;42:233-7.

18. Lee JR, Choi JY, Kim Y-D, Choi J. Laser Peripheral Iridotomy with Iridoplasty in Primary Angle Closure Suspect: Anterior Chamber Analysis by Pentacam. Korean Journal of Ophthalmology : KJO 2011;25:252-6.

19. Li X, Wang Z, Cao Q, Hu L, Tian F, Dai H. Pentacam could be a useful tool for evaluating and qualifying the anterior chamber morphology. International Journal of Clinical and Experimental Medicine 2014;7:1878-82.

20. Talajic JC, Lesk MR, Nantel-Battista M, Harasymowycz PJ. Anterior segment changes after pilocarpine and laser iridotomy for primary angle-closure suspects with Scheimpflug photography. J Glaucoma 2013;22:776-9.

21. Vryonis N, Nikita E, Vergados I, Theodossiadis P, Filippopoulos T. Anterior chamber morphology before and after laser peripheral iridotomy determined by Scheimpflug 
technology in white patients with narrow angles.

J Glaucoma 2013;22:679-83.

22.Jain R, Grewal D, Grewal SP. Quantitative analysis of anterior chamber following peripheral laser iridotomy using Pentacam in eyes with primary angle closure. Eur J Ophthalmol 2012:00.

23. Wu HJ, Ren ZQ, Bao YZ, Hou XR, Hu YW. [Evaluation the value of Pentacam arterial segment analysis system in the study of anterior segment of the eye before and after laser periphery iridectomy]. Zhonghua Yan Ke Za Zhi 2010;46:1110-4.

24. Shankar H, Taranath D, Santhirathelagan CT, Pesudovs K. Anterior segment biometry with the Pentacam: comprehensive assessment of repeatability of automated measurements. J Cataract Refract Surg 2008;34:103-13.

25.Kurita N, Mayama C, Tomidokoro A, Aihara M, Araie M. Potential of the pentacam in screening for primary angle closure and primary angle closure suspect. J Glaucoma 2009;18:506-12.

26. Antoniazzi E, Pezzotta S, Delfino A, Bianchi PE. Anterior chamber measurements taken with
Pentacam: an objective tool in laser iridotomy. Eur J Ophthalmol 2010;20:517-22.

27.Li S, Wang H, Mu D, Fu J, Wang X, Wang J, Wang N. Prospective evaluation of changes in anterior segment morphology after laser iridotomy in Chinese eyes by rotating Scheimpflug camera imaging. Clinical \& experimental ophthalmology 2010;38:10-14.

28. Pakravan M, Sharifipour F, Yazdani S, Koohestani N, Yaseri M. Scheimpflug Imaging Criteria for Identifying Eyes at High Risk of Acute Angle Closure. Journal of Ophthalmic \& Vision Research 2012;7:111-7.

29.Zou X, Duan X-C, Zhong Q. Alteration of anterior chamber in 81 glaucomatous eyes using Pentacam Scheimpflug system. International Journal of Ophthalmology 2010;3:349-51.

30. Wang N, Wu H, Fan Z. Primary angle closure glaucoma in Chinese and Western populations. Chinese medical journal 2002;115:1706-15.

31. He M, Foster P, Johnson G, Khaw P. Angleclosure glaucoma in East Asian and European people. Different diseases? Eye 2006;20:3-12. 